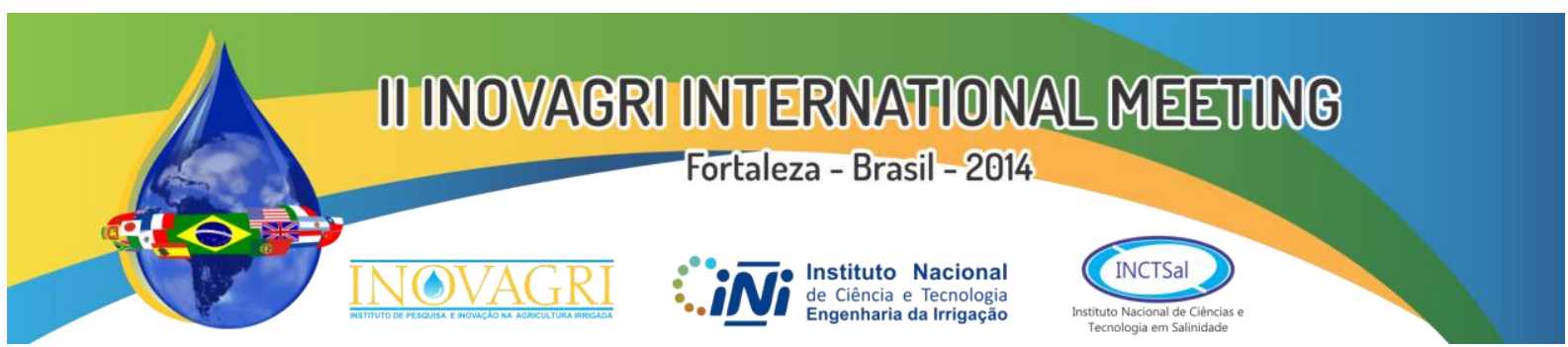

http://dx.doi.org/10.12702/ii.inovagri.2014-a167

\title{
RESPOSTA AO DEFÍCIT HÍDRICO DE CULTIVARES DE ALGODOEIRO HERBÁCEO
}

\author{
W. P. Araújo ${ }^{1}$, J. R. Pereira ${ }^{2}$, E. S. A. B. de Almeida $^{3}$, J. H. Zonta ${ }^{4}$, H. O. C. Guerra ${ }^{5}$, J. R. C. Bezerra ${ }^{4}$
}

RESUMO: Objetivando avaliar o fator de resposta ao déficit hídrico (Ky) de cultivares de algodoeiro herbáceo, um ensaio experimental foi instalado no Campo Experimental da Embrapa Algodão, no município de Barbalha, CE. Utilizou-se um delineamento com blocos casualizados, com 4 repetições, totalizando 60 subparcelas dispostas em faixas, em combinação fatorial $3 \times 5$ de três cultivares de algodoeiro herbáceo ((BRS 286 (C1),BRS Aroeira (C2) e BRS Araripe (C3)) e cinco lâminas de irrigação (260,93 (L1), 418,93 (L2), 514,21 (L3), 711,81 (L4) e 894,68 (L5)) mm, correspondente a 36, 58, 72, 100 e $126 \%$ da ETc. O algodoeiro foi plantado em fileiras simples no espaçamento de $1,00 \times 0,20 \mathrm{~m}$, com áreas por parcela de $12 \times 15 \mathrm{~m}\left(180 \mathrm{~m}^{2}\right)$, por subparcela de $12 \times 3 \mathrm{~m}\left(36 \mathrm{~m}^{2}\right)$, útil de $6 \mathrm{~m}^{2}$ e total experimental de $2.160 \mathrm{~m}^{2}$. O volume de reposição de água em cada evento de irrigação foi calculada em função da segunda faixa (3 a $6 \mathrm{~m}$ da linha central de aspersores), denominada de lâmina de controle, determinada pela evapotranspiração da cultura $\left(E T_{\mathfrak{c}}\right)$, estimada por Penman-Monteith, multiplicada pelos coeficientes da cultura do algodão $\mathrm{Kc}$ nas diferentes fases fenológicas $\left(\mathrm{ETc}=\mathrm{ET}_{0} \times \mathrm{Kc}\right)$. Para estimar Ky usou-se um parâmetro adimensional, relacionando a redução relativa no rendimento com o consumo relativo de água, segundo FAO 33. Este fator indica a tolerância de determinada cultura ao estresse hídrico. Os valores de Ky para as cultivares BRS Aroeira, BRS Araripe e BRS 286 foram iguais a 0,31; 0,46 e 0,67, respectivamente, significando que para cada unidade de decréscimo na lâmina de irrigação, encontrou-se um decréscimo de 0,67; 0,46 e 0,31 unidade na produtividade do algodoeiro BRS 286, BRS Araripe e BRS Aroeira. A cultivar de algodão que melhor respondeu ao déficit hídrico foi o BRS Aroeira.

PALAVRAS-CHAVE: Gossypium hirssutum L. r. latifolium H., rendimento, lâminas

\section{RESPONSE WATER DEFICIT COTTON CULTIVARS}

ABSTRACT: To evaluate the response factor to water deficit (Ky) of cotton cultivars, an experimental trial was installed at the Experimental Embrapa Cotton, in the municipality of Barbalha, CE, a randomized block design, with four replications, totaling 60 plots arranged in a split block scheme, in a $3 \times 5$ factorial combination of three cotton cultivars (BRS 286 (C1), BRS Aroeira (C2) and BRS Araripe (C3)) and five irrigation levels (260.93 (L1), 418.93 (L2), 514.21 (L3), 711.81 (L4) and 894.68 (L5)) mm, corresponding to $36,58,72,100$ and $126 \%$ of ETc. The cotton was planted in single rows spaced of $1.0 \times 0.20 \mathrm{~m}$, with areas per plot of $12 \times 15\left(180 \mathrm{~m}^{2}\right)$, per subplot of $12 \times 3 \mathrm{~m}\left(36 \mathrm{~m}^{2}\right)$, useless of $6 \mathrm{~m}^{2}$, and total experimental of $2160 \mathrm{~m}^{2}$. The volume of water replacement in each irrigation event was based in the second split block (3-6 m of central line sprinkler), called control level, and determined by crop evapotranspiration $\left(\mathrm{ET}_{0}\right)$, estimated by Penman-Monteith, multiplied by cotton $\mathrm{Kc}$ in its phases of development $\left(\mathrm{ET}_{\mathrm{C}}=\mathrm{ET}_{0} \mathrm{x} \mathrm{Kc}\right)$. Ky used to estimate a dimensionless parameter relating the relative reduction in yield with the relative water consumption, according to FAO 33. This factor indicates the particular culture of tolerance to water stress. Ky values for BRS Aroeira, BRS Araripe e BRS 286 and were equal to 0.31, 0.46 and 0.67, meaning that for every unit decrease in water depth, found a decrease of $0.67,0.46$ and 0.31 unit cotton yield BRS

\footnotetext{
${ }^{1}$ Doutorando, UAEAg/CTRN/UFCG. Campina Grande - Paraíba. Email: wpacordao@ hotmail.com.

2 Doutorando, UAEAg/CTRN/UFCG. Pesquisador da Embrapa Algodão. Campina Grande - Paraíba. Email: jose.r.pereira@embrapa.br.

${ }^{3}$ Eng. Agrônoma. Estagiária da Embrapa Algodão. Campina Grande - Paraíba. Email: ericasamara@ gmail.com.

4 Doutor, Pesquisador da Embrapa Algodão. Campina Grande - Paraíba. Email: joao-henrique.zonta@embrapa.br; jose.cortez-bezerra@embrapa.br.

${ }^{5}$ Professor da UAEAg/CTRN/UFCG. Campina Grande - Paraíba. Email: hugo_carvallo@ hotmail.com.
}
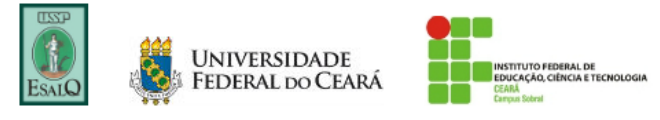
286, BRS Araripe and BRS Aroeira. A cotton cultivar that best responded to water deficit was the BRS Aroeira.

KEYWORDS: Gossypium hirssutum L. r. latifolium H., income, blades

\section{INTRODUÇÃO}

A cotonicultura brasileira, em geral, ressente-se dos baixos índices tecnológicos apresentados pelos produtores que, na maioria das vezes, são responsáveis pelas baixas produtividades, principalmente no Nordeste, onde existem limitações com relação à disponibilidade de água e de nutrientes no solo (OLIVEIRA et al., 2008).

O déficit hídrico é o resultado (negativo) do balanço hídrico em que o total de água que entra no sistema via precipitação é menor que a quantidade total de água perdida pela evaporação e pela transpiração pelas plantas (DUARTE et al., 2012).

Com base no exposto, conhecer a resposta das culturas, como por exemplo, o algodoeiro, à escassez de água (déficit hídrico), em ambientes específicos e/ou diferentes, como a região semiárido, é de grande importância para realizarmos comparações econômicas entre as opções de cultivo para a região, visto que, por outro lado, a irrigação com déficit cria um estresse hídrico na planta que pode afetar o crescimento, desenvolvimento e a produtividade das culturas.

Para avaliar o efeito do estresse hídrico na produtividade das culturas, Doorenbos \& Kassan (1979) propuseram que o efeito desse estresse poderia ser quantificado por uma função linear, onde a declividade da linha (Ky) é o fator de resposta da produtividade da cultura em função do déficit hídrico, que varia dependendo da cultura e do estágio de crescimento que a mesma sofreu estresse hídrico. Quanto mais próximo de zero este valor, mais tolerante é a cultura ao estresse hídrico. Culturas que apresentam valor de Ky maior que 1 são sensíveis ao estresse hídrico. Segundo estudo da FAO (2002), após um levantamento de diversos trabalhos que relacionam a produtividade do algodoeiro à irrigação com déficit, o valor de Ky para o algodoeiro pode variar de 0,46 a 0,99 , dependendo da época à qual a planta foi submetida ao estresse hídrico e do tipo de irrigação, o que demonstra que a cultura apresenta tolerância ao estresse hídrico, visto que o valor de Ky é menor que 1 .

Este fator é um indicativo de que a cultura é tolerante ao estresse hídrico. Quando o fator de resposta é maior que 1, indica que o decréscimo relativo na produtividade para um dado déficit na irrigação é proporcionalmente maior que o decréscimo relativo na lâmina de irrigação ou evapotranspiração (KIRDA et al., 1999). A FAO (2012) classifica os valores de Ky e diz que eles são específicos para as diferentes culturas, podendo ser classificados como: quando a resposta da cultura é muito sensível ao déficit hídrico $(\mathrm{Ky}>1)$; quando a cultura é mais tolerante ao déficit hídrico $(\mathrm{Ky}<1)$ e quando a redução da produção é diretamente proporcional ao consumo de água reduzida $(\mathrm{Ky}=1)$.

Diante do exposto, objetivou-se, com o presente trabalho, avaliar a resposta ao déficit hídrico de cultivares de algodoeiro herbáceo sob diferentes lâminas de irrigação.

\section{MATERIAL E MÉTODOS}

O experimento foi conduzido no Campo Experimental da Embrapa Algodão, no município de Barbalha, CE, localizado nas coordenadas geográficas $7^{\circ} 19^{\prime} \mathrm{S}$ de latitude, $39^{\circ} 18^{\prime} \mathrm{O}$ de longitude e 409,03 m de altitude (RAMOS et al., 2009) a aproximadamente $550 \mathrm{~km}$ da capital do Estado, situada na Mesorregião Sul Cearense e na Microrregião do Cariri Cearense, no período de julho a dezembro de 2010, período compreendido entre o plantio e a colheita (LEDO et al,. 2011).

O clima da região, de acordo com a classificação climática de Köppen adaptada ao Brasil (COELHO \& SONCIN, 1982), é do tipo "CSa", semiúmido, com verão quente e seco (4 a 5 meses) e chuvas de outono e inverno. O período chuvoso é de março a junho e o mais seco é de outubro a dezembro.

O solo da área experimental é do tipo NeossoloFlúvico e sua caracterização química, conforme Boletim No 46/11 do Laboratório de Solos da Embrapa Algodão foi à seguinte: pH de 7,5; 125,$1 ; 78 ; 7,2 ; 6,0$ e 0,0 mmolc $\mathrm{dm}^{-3}$ de cálcio, magnésio, sódio, potássio e alumínio, respectivamente; $24,5 \mathrm{mg} \mathrm{dm}^{-3}$ de fósforo e $18,8 \mathrm{~g} \mathrm{~kg}^{-1}$ de matéria orgânica. 
O solo da área experimental foi preparado 15 dias antes do plantio, por meio de arações com arado escarificador a uma profundidade de $20 \mathrm{~cm}$, seguidas de duas gradagens com grade niveladora.

$\mathrm{Na}$ adubação foram aplicados 90,60 e $20 \mathrm{~kg} \mathrm{ha}^{-1}$ de $\mathrm{N}, \mathrm{P}$ e K, respectivamente, sendo o $\mathrm{N}$ parcelado em três vezes, aplicadas na forma de uréia, com aplicações de 10, 40 e $40 \%$ na adubação de fundação, primeira e segunda adubações de cobertura. $\mathrm{O} \mathrm{N}$ e $\mathrm{P}$ foram aplicados na forma de MAP (fosfato monoamônico) e o K na forma de cloreto de potássio (KCL).Para controle de plantas daninhas, foram feitas três capinas manuais à enxada.

Os tratamentos consistiram da combinação fatorial $3 \times 5$ de três cultivares de algodoeiro herbáceo ((BRS 286 (C1); BRS Aroeira (C2) e BRS Araripe (C3)) e cinco lâminas de irrigação, 260,93 (L1), 418,93 (L2), 514,21 (L3), 711,81 (L4) e 894,68 (L5) mm.

Combinados, os fatores resultaram em 15 tratamentos: (1.L5C1), (2.L5C2), (3.L5C3), (4.L4C1), (5.L4C2), (6.L4C3), (7.L3C1), (8.L3C2), (9.L3C3), (10.L2C1), (11.L2C2), (12.L2C3), (13.L1C1), (14.L1C2) e (15.L1C3), em delineamento em blocos casualizados, com 4 repetições, totalizando 60 subparcelas dispostas em faixas.

As cultivares de algodoeiro herbáceo foram plantadas em fileiras simples com espaçamento de $1,0 \times 0,20 \mathrm{~m}$, tendo a área de cada parcela experimental $12 \times 15 \mathrm{~m}$ perfazendo uma área de $180 \mathrm{~m}^{2}$ por parcela, e área das subparcelas de $12 \times 3 \mathrm{~m}$, com área de $36 \mathrm{~m}^{2}$ e uma área útil (duas fileiras centrais) de $6 \mathrm{~m}^{2}$; portanto, com área total experimental de $2160 \mathrm{~m}^{2}$.

Imediatamente antes do plantio foi efetuada uma irrigação em toda a área de modo a levar o solo à capacidade de campo, e após o plantio, a cada 4 dias uma irrigação com pequena lâmina, de modo a assegurar a boa germinação das sementes. A partir do estabelecimento da cultura, as irrigações foram efetuadas uma vez por semana.

O volume de reposição de água foi determinado na segunda faixa, correspondente a segunda subparcela, denominada de lâmina de controle, e calculado em função da evapotranspiração da cultura, determinada multiplicando a evapotranspiração de referência, $\mathrm{ET}_{0}$, pelo coeficiente da cultura do algodão $\mathrm{Kc}\left(\mathrm{ET}_{0} \mathrm{x} \mathrm{Kc}\right)$. A evapotranspiração de referência $\left(\mathrm{ET}_{0}\right)$, foi calculada pelo método de Penman-Monteith (ALLEN et al., 2006) e os coeficientes de cultivo conforme Bezerra et al. (2008) de 0,76 (5 a 20 DAP) para a fase I, de 0,95 (21-41 DAP) para a fase II, de 1,09 (42-82 DAP) para a fase III e de 0,88 (83-103 DAP) para a fase IV.Os dados climáticos para uso no cálculo da $\mathrm{ET}_{0}$ foram obtidos da Estação Meteorológica Automática do Instituto Nacional de Meteorologia - INMET de Barbalha, CE.

A água utilizada na irrigação foi de um poço artesiano localizado próximo ao experimento. Antes do início do experimento uma amostra da água foi retirada e levada ao Laboratório de Análises de Solos e Nutrição de Plantas da Embrapa Algodão, para determinações de suas características químicas e classificação, apresentando salinidade média e baixa concentração de sódio podendo ser usada para irrigação sempre que houver um grau moderado de lixiviação e cuidados especiais no manejo do solo.

As lâminas de irrigação aplicadas em cada tratamento ao longo do experimento $(260,93$, $418,93,514,21,711,81$ e $894,68 \mathrm{~mm}$ ) representam respectivamente, $36,58,72,100$ e $125 \%$ da evapotranspiração da cultura (ETc).

Para avaliar a resposta da cultura ao déficit hídrico usou-se um parâmetro adimensional que relaciona a redução relativa no rendimento com o consumo relativo de água, segundo FAO 33 (DOORENBOS \& KASSAM, 1979), sendo:

$$
1-\mathrm{Ya} / \mathrm{Ym}=\mathrm{Ky}(1-\mathrm{La} / \mathrm{Lm})(1)
$$

em que:

Ya é a produtividade em dada lâmina de irrigação, $\mathrm{kg} \mathrm{ha}^{-1}$;

Ym é a produtividade máxima, $\mathrm{kg} \mathrm{ha}^{-1}$;

$\mathrm{Ya} / \mathrm{Ym}$ é a produtividade relativa, adimensional;

1 - (Ya/Ym) é o decréscimo na produtividade relativa, adimensional;

La é a lâmina de irrigação aplicada que acarreta na produtividade Ya, mm;

Lm é a lâmina máxima de irrigação aplicada, mm;

La/Lm é a lâmina de irrigação relativa, adimensional; 
1 - (La/Lm) é o decréscimo relativo na lâmina de irrigação aplicada, adimensional;

Ky é o fator de resposta definido como o decréscimo na produtividade com relação ao decréscimo da lâmina de irrigação aplicada, adimensional.

\section{RESULTADOS E DISCUSSÃO}

Na Tabela 1 são apresentados os dados com relação ao fator de resposta do rendimento(Ky) de três cultivares de algodoeiro herbáceo sob diferentes lâminas de irrigação.

Os valores do fator ky para o algodoeiro BRS Araripe, BRS Aroeira e BRS 286 foram, respectivamente, 0,35; 0,19 e 0,18 para a lâmina de irrigação aplicada de 711,81 (L4) mm, sendo 894,68 (L5) mm considerados a lâmina de máxima produtividade para todas as cultivares estudada (Tabela 1). A lâmina de maior déficit hídrico (L1 - 260,93 mm) não foi á lâmina que apresentou menor valor para o fator ky para todas as cultivares estudadas. Observa-se que a lâmina que apresentou menor valor do fator ky foi a de 514,21 (L3) mm $(0,07)$ para a BRS Araripe, de 418,93 (L2) e 514,21 (L3) $\mathrm{mm}$ ((valores negativos devido a produtividade de essas lâminas ter sido maior que as das lâminas de 711, 81 (L4) e 894,68 (L5) mm)) para a BRS Aroeira e de 514,21 (L3) mm (0,22) para a BRS 286 (Tabela 1).

Em trabalho realizado no semiárido da Índia, Singh et al. (2010) encontraram valores próximos aos do presente trabalho com ky de 1,02;0,98 e 0,28 com níveis de irrigação de 50, 60 e $90 \%$ da ETc.

Na Figura 1 é apresentado o gráfico que relaciona a diminuição na produtividade da cultura em função da diminuição da lâmina de irrigação, ou seja, obtendo-se um valor sazonal de ky para as três cultivares de algodoeiro herbáceo estudadas.

Observa-se que os valores de ky para as cultivares de algodoeiro herbáceo BRS Aroeira, BRS Araripe e BRS 286 foram, respectivamente iguais a 0,3155; 0,4672 e 0,6705, ou seja, nas condições em que o estudo foi realizado, para cada unidade de decréscimo na lâmina de irrigação, encontra-se um decréscimo de 0,$6705 ; 0,4672$ e 0,3155 unidades na produtividade do algodoeiro BRS 286, BRS Araripe e BRS Aroeira, respectivamente. O ky, menor que 1, indica que as cultivares estudadas são mais tolerantes ao déficit hídrico (FAO, 2012).

De maneira geral, a cultivar que melhor respondeu ao déficit hídrico foi o algodoeiro herbáceo BRS Aroeira (menor declividade na curva), seguida das BRS Araripe e BRS 286, pois, quanto mais próximo de zero for o valor do ky maior é a resistência da cultivar quanto ao déficit hídrico. Para cada 50\% no decréscimo da lâmina de irrigação aplicada, ocorreram decréscimos estimados próximos de 14; 22 e 33\% na produtividade do algodoeiro BRS Aroeira, BRS Araripe e BRS 286 (Figura 1).

Valores superiores aos do presente trabalho foram encontrados no semiárido da Turquia, por Dagdelen et al. (2006) e Dagdelen et al. (2009), com valores de ky variando 0,78 - 0,98. Para a produção de sementes de algodão na planície de Seyhan, Ertek \& Kanber (2003) sugeriram fatores ky entre $0,38-0,84$, em que os valores para as três cultivares estudadas se encontram nesta faixa; já segundo a FAO (2012) o valor sazonal do ky para a cultura do algodão é de 0,85 .

\section{CONCLUSÕES}

As cultivares estudadas apresenta tolerância ao déficit hídrico sendo a BRS Aroeira a mais tolerante, seguida das BRS Araripe e BRS 286.

\section{REFERÊNCIAS BIBLIOGRÁFICAS}

ALLEN, R. G.; PRUIT, W. O.; WRIGHT, J. L.; HOWELL, T. A.; VENTURA, F.; SNYDER, R.; ITENFISU, D.; STEDUTO, P.; BERENGENA, J.; YRISARRY, J. B.; SMITH, M.; PEREIRA, L. S.; RAES, D.; PERRIER, A.; ALVES, I.; WALTER, I.; ELLIOTT, R. A recommendation on standardized surface resistance for hourly calculation of reference ETo by the FAO56 PenmanMonteith method. AgriculturalWater Management, Amsterdam, v. 81, p. 1-22, 2006. http://dx.doi.org/10.1016/j.agwat.2005.03.007 
BEZERRA, J. R. C.; LUZ, M. J. da S. e.; BARRETO, A. N.; AMORIM NETO, M. da S.; SILVA, L. C. Irrigação do algodoeiro herbáceo. In: BELTRÃO, N. E. de M.; AZEVEDO, D. M. P. de. O agronegócio do algodão no Brasil. Brasília: Embrapa Informação Tecnológica, 2008. v.2, Cap.27, p.875-950.

COELHO, M. A.; SONCIN, N. B. Geografia do Brasil. São Paulo: Moderna. 1982. 368p.

DAGDELEN, N.; BASAL, H.; YILMAZ, E.; GURBZ, T.; AKCAY, S. Different drip irrigation regimes affect cotton yield, water use efficiency and fiber quality in western Turkey. Agricultural Water Management, v.96, n.1, p.111-120, 2009. http://dx.doi.org/10.1016/j.agwat.2008.07.003

DAGDELEN, N.; YILMAZ, E.; SEZGIN, F.; GÜRBÜZ, T. Water-yield relation and water use efficiency of cotton (Gossypiumhirsutum L.) and second crop (Zea mays L) in western Turkey. $\begin{array}{lllll}\text { Agricultural Water } & \text { Management, } & \text { v. } & \text { 82, 63-85, }\end{array}$ http://dx.doi.org/10.1016/j.agwat.2005.05.006

DOORENBOS, J., KASSAM, A. H., Yield response to water. FAO Irrigation and Drainage Paper NO.33, FAO, Rome, Italy.1979.

DUARTE, J. M. de L; LIMA, A. D; NASCIMENTO, R. S; VIANA, T. V. de A; SARAIVA, K. R; AZEVEDO, B. M. de. Eficiência do uso da água na produção de óleo do girassol (helliantusannuusL.), sob suspensão hídrica. RevistaBrasileira de AgriculturaIrrigada, v.6, no .3, p. $166-175,2012$.

ERTEK, A.; KANBER, R. Effects of different drip irrigation programs on the boll number and shedding percentage and yield of cotton. Agricultural Water Management, 60, n.1, p.1-11, 2003. http://dx.doi.org/10.1016/S0378-3774(02)00156-7

FAO. Deficit Irrigation Practices. FAO Water Reports NO. 22, FAO, Rome, Italy. 2002.

FAO. Crop yield response to water. FAO Water Reports NO. 66, FAO, Rome, Italy.2012.

KIRDA, C.; MOUTONNET, P.; HERA, C.; NIELSEN, D. R. (Eds.). Crop Yield Response to

Deficit Irrigation. Kluwer Academic Publishers, Dordrecht, The Netherlands. 1999. http://dx.doi.org/10.1007/978-94-011-4752-1

LEDO, E. R. F.; SILVA, M. G. da; NOGUEIRA, D. H.; MIRANDA, E. P. de; ARAÚJO, E. M.; ARAÚJO, E. M. Avaliação de metodologias empregadas no cálculo da temperatura média diária do ar na região de Barbalha-CE. Revista Brasileira de Agricultura Irrigada, v.5, n.4, p.310 - 319, Fortaleza, CE, 2011.

OLIVEIRA, F. A. de; SILVA, M. N. B. da S.; OLIVEIRA, A. P. de; SANTOS, D.; PEREIRA, W. E.; OLIVEIRA, R. C. de; GONDIM, S. C. Efeito da irrigação e da adubação nitrogenada sobre algumas características de desenvolvimento do algodão colorido verde. Revista brasileira de oleaginosas e fibrosas, v.12, n.1, p. 49-57, jan./abr. 2008.

RAMOS, A. M.; SANTOS, L. A. R. dos; FORTES, L. T. G (Orgs.) Normas climatológicas do Brasil: 1961-1990. Brasília: INMET, 2009. 465p.

SINGH, Y.; RAO, S. S.; REGAR, P. L. Deficit irrigation and nitrogen effects on seed cotton yield, water productivity and yield response factor in shallow soils of semi-arid environment. $\begin{array}{llll}\text { AgriculturalWater } \quad \text { Management, } & \text { v.97, } & \text { p.965-970, }\end{array}$ http://dx.doi.org/10.1016/j.agwat.2010.01.028 


\section{W. P. Araújo et al.}

Tabela 1. Relação entre o decréscimo no rendimento relativo 1- (Ya/Ym), decréscimo relativo na lâmina aplicada (1- La/Lm) e o fator de resposta do rendimento $(\mathrm{Ky})$ de três cultivares de algodoeiro herbáceo submetidos a diferentes lâminas de irrigação, Barbalha, CE, 2010.

\begin{tabular}{lccc}
\hline BRS Araripe & $1-(\mathrm{Ya} / \mathrm{Ym})$ & $1-(\mathrm{La} / \mathrm{Lm})$ & $\mathrm{Ky}$ \\
\hline $260,93 \mathrm{~mm}$ & 0,49 & 0,70 & 0,69 \\
$418,93 \mathrm{~mm}$ & 0,17 & 0,53 & 0,32 \\
$514,21 \mathrm{~mm}$ & 0,03 & 0,42 & 0,07 \\
$711,81 \mathrm{~mm}$ & 0,07 & 0,20 & 0,35 \\
$894,68 \mathrm{~mm}$ & 0 & 0 & 0 \\
\hline BRS Aroeira & & & 0,32 \\
\hline $260,93 \mathrm{~mm}$ & 0,23 & 0,70 & - \\
$418,93 \mathrm{~mm}$ & - & - & - \\
$514,21 \mathrm{~mm}$ & - & - & 0,19 \\
$711,81 \mathrm{~mm}$ & 0,04 & 0,20 & 0 \\
$894,68 \mathrm{~mm}$ & 0 & 0 & \\
\hline BRS 286 & & & 0,86 \\
\hline $260,93 \mathrm{~mm}$ & 0,60 & 0,70 & 0,69 \\
$418,93 \mathrm{~mm}$ & 0,36 & 0,53 & 0,22 \\
$514,21 \mathrm{~mm}$ & 0,09 & 0,42 & 0,18 \\
$711,81 \mathrm{~mm}$ & 0,03 & 0,20 & 0 \\
$894,68 \mathrm{~mm}$ & 0 & 0 & \\
\hline
\end{tabular}

\section{Decréscimo na lâmina de irrigação}

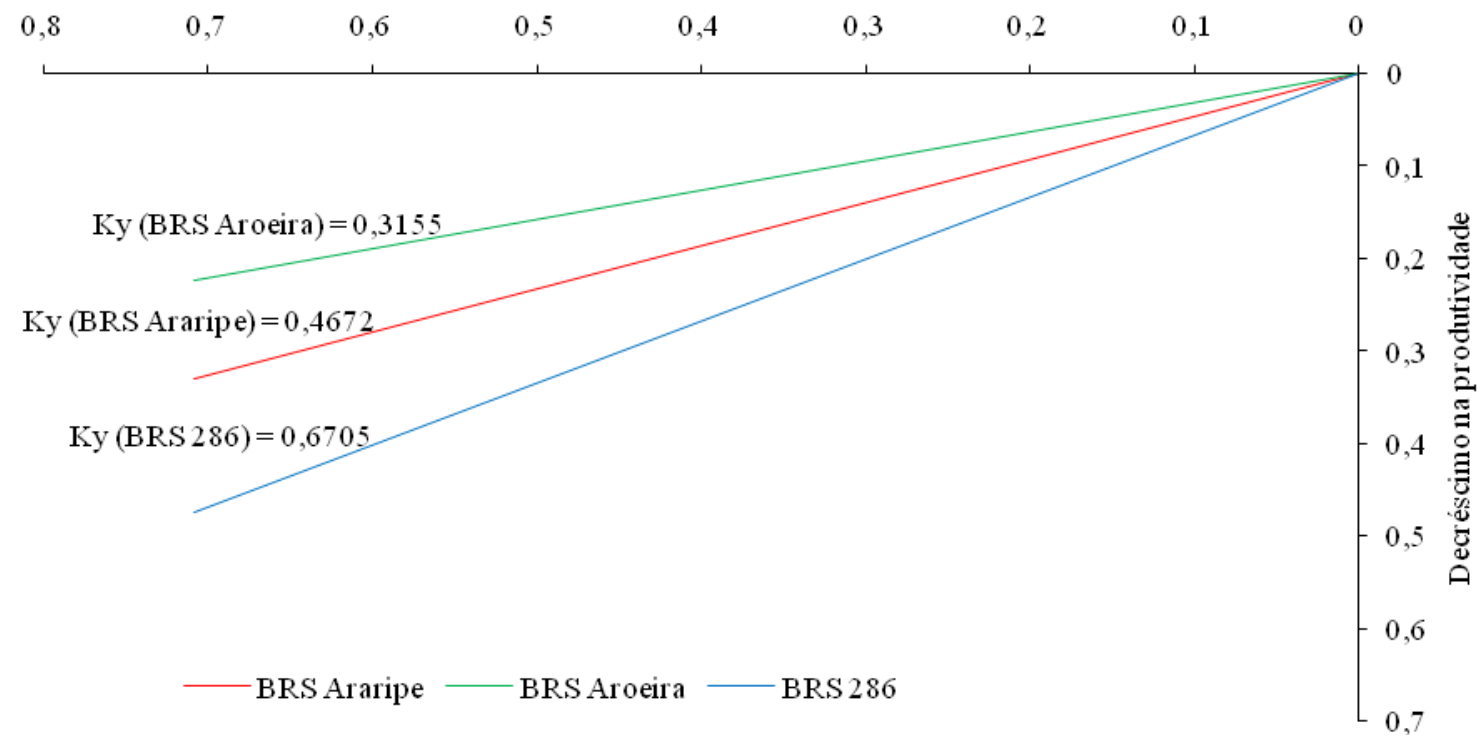

Figura 1. Resposta relativa da produtividade de três cultivares de algodoeiro herbáceo submetidos a diferentes lâminas de irrigação ao relativo déficit hídrico, Barbalha, CE, 2010. 